\section{Assessment of Bayesian Estimators for Osteoporosis Analysis}

Leandro Luiz Mazzuchello1, Larissa Letieli Toniazzo de Abreu', Carolina Pedrassani de Lira1, Maitê Gabriel dos Passos', Ramon Venson', Abigail Lopes ${ }^{1,2}$, Diego Garcia1, Maria Marlene de Souza Pires ${ }^{3}$, Eros Comunello4, Luciane Bisognin Ceretta ${ }^{2}$, Paulo João Martins ${ }^{1}$, Priscyla Waleska Simões ${ }^{1,2}$

\section{Abstract}

Background: Bayesian classifiers have the advantage of determining the class to which a given record belongs compared to traditional classifiers, taking as base the probability of an element belonging to a class. Thus, the diagnosis of diseases such as osteoporosis and osteopenia can become faster and precise. This paper presents an assessment of the accuracy of the Bayesian classifiers Bayes Net, Naive Bayes and Averaged One-Dependence Estimators to support diagnoses of osteopenia and osteoporosis.

Method: The methodology that guided the development of this research relied on the choice of database, the study of the Bayes Net, Naive Bayes and Averaged One-Dependence Estimators algorithms, and the description of the experiments.

Results: The algorithm with the highest specificity was Bayes Net, $(53.0 \pm 0.27)$. The highest accuracy was obtained using the AODE classifier $(83.0 \pm 0.17)$. Our results showed higher mean instances correctly classified using the Naive Bayes algorithm (82.84 \pm 14.42$)$, and the average of incorrectly classified instances was higher for Bayes Net (17.46 \pm 14.76$)$.

Conclusion: Based on the statistical measures analyzed in the experiments (instances classified correctly and incorrectly, the kappa coefficient, mean absolute error, sensitivity, specificity, accuracy, recall, F-measure, and Area Under Curve (AUC)), all classifiers showed good results, thus, given these data, it is possible to produce a reasonably accurate estimate of the diagnosis.
1 Research Group in Information and Communications Technology in Health, Universidade do Extremo Sul Catarinense (UNESC), Criciúma, SC, Brazil.

2 Graduate Program in Public Health (PPGSCol). Universidade do Extremo Sul Catarinense (UNESC), Criciúma, SC, Brazil.

3 Graduate Program in Medical Sciences (PPGCM/UFSC). Universidade Federal de Santa Catarina, Florianópolis (UFSC), SC, Brazil.

4 INCOD - National Institute for Digital Convergence. Universidade Federal de Santa Catarina, Florianópolis (UFSC), SC, Brazil.

\section{Contact information:}

\section{Priscyla Waleska Simões.}

Research Group in Information and Communication Technology in Health, Graduate Program in Public Health (PPGSCol).

Address: Universidade do Extremo Sul Catarinense (UNESC). Av. Universitária, 1105 - Criciúma - SC - Bairro Universitário, CEP - 88806-000.

झ pri@unesc.net

\section{Keywords}

Medical Informatics; Artificial Intelligence; Data Mining; Bayes Theorem; Osteoporosis. 


\section{Introduction}

Osteoporosis is a disease that results in the decline in the health of the world population and challenges the public health and medical professions. It is important to focus on this disease due to the morbidity and mortality resulting from osteoporotic fractures, yet the majority of patients suffering from this condition remain untreated: less than $20 \%$ of individuals with fragile bone fractures receive adequate therapy [1].

Osteoporosis is characterized by low bone density and microarchitectural deterioration of bone tissue, leading to increased skeletal fragility and increased risk of fractures, particularly in the backbone, hip, forearm, and humerus [2]. Although the risk of fractures increases due to decreased bone density, most fractures occur in patients with osteopenia because the majority of the population is classified as having this disease [3]. Osteopenia is a skeletal disorder defined according to the criteria of the World Health Organization (WHO) by a decreased bone mineral density t-score between -1 and -2.54 for either the spine or hip [4].

The incidence of osteoporosis is increasing due to the aging population, ranging from $14 \%$ to $29 \%$ in women over 50 years of age and affecting up to $70 \%$ of women over 80 years old. However, in Brazil, there are no studies that definitively determine the incidence of the disease because the racial integration and heterogeneity of the regions suggest different risk factors [2].

This disease is a major cause of morbidity and mortality in the elderly. Although less common in men than in women, more than 8 million men in the United States have osteoporosis [5], and the mortality rate for hip fractures (as well as other vertebral fractures) is higher in males.

Data mining (DM) offers methods and tools based on statistical concepts and artificial intelligence machines capable of assisting in the construction of more significant knowledge. DM is an automated discovery process to find relevant and implicit information in large databases. DM techniques attempt to discover patterns and useful rules that are otherwise small or imperceptible and can aid the researcher in their evaluation [6], thus allowing differentiated analyses of the data, the development of predictive models and the formulation of hypotheses.

The tasks related to DM include a classification system to establish a function that maps a set of elements to a number of classes. In recent years, this system has been the most utilized tool in health care [7]. Therefore, to address the uncertainty inherent in biomedical diagnoses, Bayesian classifiers, which are based on statistical models, have the advantage of determining the class to which a given record belongs compared to traditional classifiers, taking as base the probability of an element belonging to a class. Thus, the diagnosis of diseases such as osteoporosis and osteopenia can become faster and precise [8].

This paper presents an evaluation of the accuracy of the Bayesian classifiers Bayes net (BN), Naive Bayes (NB) and Averaged One-Dependence Estimators (AODE) to support the diagnosis of osteopenia and osteoporosis.

\section{Methods}

Detailed below is the methodology that guided the development of this research, including the choice of the experiments.

The database used in the experiments originated from a transverse study wih a consecutive sample involving 1871 women in southern Santa Catarina who were subjected to bone densitometry measurements under a specialized service from 2010 to 2012. This study was approved by the Ethics Committee under protocol 82939/2012. The data collected contain census characteristics because the subjects represented all of the different ethnic groups within the general population. The service consulted provides patient care through the Brazilian Unified Health System and private plans. 
The variables used in the experiments are presented in Table 1.

Table 1. Variables used in the experiments.

\begin{tabular}{|c|c|}
\hline Description & $\begin{array}{c}\text { Attributes - } \\
\text { preprocessed database }\end{array}$ \\
\hline Dichotomous Fracture & 1-Yes, 2-No \\
\hline Femur Dichotomous & 1-Yes, 2-No \\
\hline Dichotomous Column & 1-Yes, 2-No \\
\hline Forearm Dichotomous & 1-Yes, 2-No \\
\hline Costa Dichotomous & 1-Yes, 2-No \\
\hline Menopause Dichotomous & 1-Yes, 2-No \\
\hline Calcium Dichotomous & 1-Yes, 2-No \\
\hline TRH Dichotomous & 1-Yes, 2-No \\
\hline Oophorectomy Dichotomous & 1-Yes, 2-No \\
\hline Rape of vulnerable & $1.64(1.10-2.45)$ \\
\hline Hysterectomy Dichotomous & 1-Yes, 2-No \\
\hline Dichotomous Average Age & 1- 49 or younger, 2- 50 \\
\hline Dichotomized To 25 And IMC & $1-25$ or younger \\
\hline 25 Or More & 2- 26 or older \\
\hline Thyroid Medication For & 1-Yes, 2-No \\
\hline Dichotomous Outcome & $\begin{array}{c}\text { 1-normal, 2- osteopenia } \\
\text { and osteoporosis }\end{array}$ \\
\hline
\end{tabular}

The Bayesian classifiers used in this research are based on the Bayes theorem, which calculates the probability of an instance belonging to a certain pre-determined class. This instance is assigned to the class with the highest probability after applying the Bayes theorem [6].

Bayesian classification has certain advantages over classical classification because the graph structure is fixed, and the values are unconditionally independent on each other such that the classifier used to estimate the parameters implied by the algorithm becomes simple because the dependency ratio is fixed and reduced by two variables -thus, only numerical parameters can be learned [9]. The algorithms used in the tests were Naive Bayes, Averaged One-Dependence Estimators and Bayes Net and are described below. a) Naive Bayes: The NB learns the conditional probability of each data attribute belonging to a particular class from a sample of data. Then, given a new case, this algorithm uses the Bayes rule to calculate the conditional probability of the class to which the element belongs given a set of attributes and select the value of the class with the highest probability a posteriori [10]. It is considered the most efficient classifier because its main features are simplicity and precision, and although its structure is always fixed, some studies have shown high accuracy and low error rates associated with the NB [11].

b) Averaged One-Dependence Estimators: This algorithm is considered an improvement on the NB and an interesting alternative to other semi-naive approaches. It provides a good trade-off between efficiency and performance. To maintain efficiency, the AODE is restricted to the exclusive use of one estimator for the dependency relationship. Specifically, the AODE can be considered as an ensemble of SuperParent One-Dependence Estimators (SPODEs) because every attribute depends on the class and another shared attribute, which is designated as the superparent [12].

c) Bayes Net: This algorithm belongs to a family of constraint-based algorithms that use conditional independence tests (based on information measures) to decide if a pair of nodes is to be connected or disconnected [13]. The differences of Bayes-Net with respect to its relatives are the use of the Bonferroni correction to adjust the threshold used in every statistical test, the depth of the independence tests (i.e., how many variables are considered in the conditional set), and the inclusion of a parameter to control the significant percentage of information gain [13].

The experiments included the following steps: data cleaning and binarization; transformation of 
variables; selection tool; data mining; variable selection and use of classifiers.

In data cleaning and binarization, records with inconsistent information were either corrected or deleted (if missing information); however, it was found that some of the variables associated with the diagnosis were already binarized (according to medical specialist). In the transformation of variables, fields containing spaces were replaced by underlines and empty fields by question marks.

The Weka shell was chosen for the experiments that pulled data from studies conducted with the Tanagra and Knime tools, as Weka provides better measures than the others for evaluating the knowledge generated.

The original database was developed using the Statistical Package software for Social Sciences (SPSS) (.sav format) and exported to .cSv format for preprocessing performed using Excel. After the preprocessing step, the Excel database (.csv) was exported to .arff format (used in the Weka tool) for data mining, which included the use of the Bayesian classifiers Naive Bayes, Bayes Net and AODE.

\section{Results}

Approximately 327 experiments were performed and selected for evaluation. The Weka tool was used to evaluate the accuracy, the correct and/ or incorrect classification of instances, the kappa coefficient, the mean absolute error, sensitivity, specificity, recall, F-measure, Area Under Curve (AUC) ROC, algorithm execution time and overall accuracy.

Our results showed higher mean instances correctly classified using the Naive Bayes algorithm $(82.84 \pm 14.42)$, and the average of incorrectly classified instances was higher for Bayes Net $(17.46 \pm 14.76)$. The result for the kappa coefficient, related to the agreement between the result of the classifier and the result presented in the database by the specialist, was higher for the Bayes Net algorithm $(0.33 \pm 0.29)$.
The lowest mean absolute error was demonstrated with the AODE algorithm $(0.18 \pm 0.14)$, which also showed higher sensitivity $(84.0 \pm 0.14)$. However, the algorithm with the highest specificity was Bayes Net, (53.0 \pm 0.27$)$. The highest accuracy was obtained using the AODE classifier $(83.0 \pm 0.17)$. All evaluation measures considered are presented in Table 2.

\section{Discussion}

Bayesian classifiers are based on Bayes' theorem and use the fundamentals of conditional probability in the classification task. Thus, they calculate the probability of an instance belonging to each predetermined class, and instance is assigned to the class with the highest probability after application of the theorem [6].

Osteoporosis, osteopenia and osteoporotic fractures generate high health care costs, which has a significant impact on public health, cause high morbidity and mortality of the affected population and vary considerably among different countries, especially due to population differences and available resources for the prevention and treatment of these diseases [14].

Several strategies for the prevention and treatment of pathological fractures are available; however, the optimal approach to classify individuals at high risk who would benefit from improved therapies remains a challenge [15]. In this context, the DM can render this process faster and more accurate, enabling therapeutic treatment at the early stages of bone degradation.

Our study showed good sensitivity measures, specificity and accuracy for the three classifiers analyzed, with AODE showing the highest accuracy (83.0) and sensitivity (84.0), followed by Naive Bayes, which had an accuracy of 82.0 and sensitivity of 83.0.

In this context, a study on neonatal jaundice performed in 2011 by the Department of Obstetrics in 
Table 2. Comparison of measures for evaluation

\begin{tabular}{|c|c|c|c|c|c|c|}
\hline Description & Algorithm & Average & $\mathrm{Cl}$ & SD & Minimum & Maximum \\
\hline \multirow[t]{3}{*}{ Instances classified correctly } & Bayes Net & 82.54 & $79.53-85.54$ & 14.76 & 41.04 & 99.67 \\
\hline & Naive Bayes & 82.84 & $79.97-85.72$ & 14.42 & 41.20 & 99.57 \\
\hline & AODE & 84.52 & $81.69-87.34$ & 14.08 & 42.81 & 99.84 \\
\hline \multirow[t]{3}{*}{ Instances classified incorrectly } & Bayes Net & 17.46 & $14.45-20.47$ & 14.76 & 0.32 & 58.95 \\
\hline & Naive Bayes & 17.15 & $14.27-20.03$ & 14.42 & 0.43 & 58.79 \\
\hline & AODE & 15.48 & $12.66-18.30$ & 14.08 & 0.16 & 57.19 \\
\hline \multirow[t]{3}{*}{ Kappa } & Bayes Net & 0.33 & $0.27-0.40$ & 0.28 & 0.00 & 0.95 \\
\hline & Naive Bayes & 0.31 & $0.26-0.37$ & 0.28 & 0.00 & 0.96 \\
\hline & AODE & 0.34 & $0.28-0.40$ & 0.29 & -0.01 & 0.97 \\
\hline \multirow[t]{3}{*}{ Medium-absolute error } & Bayes Net & 0.19 & $0.16-0.22$ & 0.14 & 0.00 & 0.43 \\
\hline & Naive Bayes & 0.19 & $0.16-0.22$ & 0.14 & 0.01 & 0.43 \\
\hline & AODE & 0.18 & $0.15-0.21$ & 0.14 & 0.00 & 0.43 \\
\hline \multirow[t]{3}{*}{ Sensitivity } & Bayes Net & 0.82 & $0.79-0.85$ & 0.15 & 0.41 & 0.80 \\
\hline & Naive Bayes & 0.83 & $0.80-0.86$ & 0.14 & 0.41 & 0.99 \\
\hline & AODE & 0.84 & $0.82-0.87$ & 0.14 & 0.43 & 0.99 \\
\hline \multirow[t]{3}{*}{ Specificity } & Bayes Net & 0.53 & $0.48-0.59$ & 0.27 & 0.00 & 0.95 \\
\hline & Naive Bayes & 0.51 & $0.46-0.57$ & 0.27 & 0.00 & 0.96 \\
\hline & AODE & 0.49 & $0.43-0.55$ & 0.29 & 0.00 & 0.95 \\
\hline \multirow[t]{3}{*}{ Accuracy } & Bayes Net & 0.81 & $0.78-0.85$ & 0.16 & 0.38 & 0.99 \\
\hline & Naive Bayes & 0.82 & $0.78-0.85$ & 0.16 & 0.37 & 0.99 \\
\hline & AODE & 0.83 & $0.79-0.86$ & 0.17 & 0.12 & 0.99 \\
\hline \multirow[t]{3}{*}{ Recall } & Bayes Net & 0.82 & $0.79-0.85$ & 0.15 & 0.41 & 0.99 \\
\hline & Naive Bayes & 0.83 & $0.80-0.86$ & 0.14 & 0.41 & 0.99 \\
\hline & AODE & 0.84 & $0.82-0.87$ & 0.14 & 0.42 & 0.99 \\
\hline \multirow[t]{3}{*}{ Measure-F } & Bayes Net & 0.81 & $0.78-0.84$ & 0.16 & 0.38 & 0.99 \\
\hline & Naive Bayes & 0.81 & $0.78-0.84$ & 0.16 & 0.37 & 0.99 \\
\hline & AODE & 0.83 & $0.80-0.86$ & 0.15 & 0.39 & 0.99 \\
\hline \multirow[t]{3}{*}{ AUC } & Bayes Net & 0.79 & $0.75-0.82$ & 0.17 & 0.04 & 0.99 \\
\hline & Naive Bayes & 0.79 & $0.76-0.89$ & 0.17 & 0.04 & 0.99 \\
\hline & AODE & 0.81 & $0.78-0.85$ & 0.16 & 0.04 & 0.99 \\
\hline \multirow[t]{3}{*}{ Time } & Bayes Net & 0.02 & $0.01-0.02$ & 0.02 & 0.00 & 0.12 \\
\hline & Naive Bayes & 0.00 & $0.00-0.00$ & 0.01 & 0.00 & 0.04 \\
\hline & AODE & 0.00 & $0.00-0.01$ & 0.01 & 0.00 & 0.04 \\
\hline
\end{tabular}


a hospital in Portugal evaluated the $\mathrm{J} 48$ classifiers, simple CART, Naive Bayes, Multilayer Perceptron, Sequential minimal optimization (SMO) and regression logistics [16]. The most accurate algorithm identified was Naive Bayes (88.0).

Similar rates were also achieved in our study regarding the Naive Bayes algorithm. One suggested explanation for the acceptable performance of this classifier is the model's ability to assign the correct class to a test observation provided that the probability of belonging to this class is greater than the probability of belonging to another class or classes, even though the probability estimates may not be accurate [17].

This algorithm was also used in a study conducted at the University of South Carolina (USA) based on the early diagnosis of melanoma [18]. In this stu$\mathrm{dy}$, they considered three classifiers - artificial neural networks, Naive Bayes, and the nearest neighbor algorithm. The Naive Bayes algorithm achieved the highest accuracy, sensitivity and specificity (both 89\%) for the outcome considered. The diagnostic accuracy of the algorithm is based on a simple Bayesian classifier, in which all attributes are independent of each other; in other words, no attribute influences any other one [19].

Considering the use of the Bayes Net classifier, a study of breast cancer data, skin diseases and diseases of the spine at the University of Wisconsin (USA) [20], evaluated (in addition to the algorithm mentioned above) the logistic model tree and showed higher accuracy associated with Bayes Net (97.0).

The applicability of Bayesian classifiers such as the Bayes Net algorithm is not restricted only to health but encompasses several areas, as shown in research on the prediction of academic dropout at the University of Rio de Janeiro, suggesting sufficient accuracy in the use of the Bayes Net algorithm (89.0) [21]. Some authors attribute the evaluation measures of this algorithm to the fact that the classifier does not allow interactions between attributes, a characteristic that can provide greater wealth in modeling and understanding of the phenomenon under study [13].

In the case of Bayes Net, the Bayesian network is constructed by either adding or deleting arcs, considering only individual pairs in relation to compliance of the attributes; that is, the rest of the nodes are not considered in analysis [13]. This reason is why the information gain measures used in Bayes Net are referred to as "local"; in other words, the network construction does not depend on a function that evaluates the entire network each time a request for either insertion or removal of nodes or arcs is created. This feature is advantageous when there are a large number of attributes, because global scoring functions cannot minimize error sites associated with the performance of the resulting network classification. Although this algorithm has good efficiency, it is not a good classifier [13].

Furthermore, one advantage of Bayes Net over Naive Bayes is that the former performs a correct subset feature selection and omits variables that are not significant from the analysis. Additionally, Bayes Net does not make the strong assumption made by Naive Bayes that the variables are conditionally independent given the class variable [13].

AODE, which had the highest accuracy in our experiments, was also the most accurate classifier in the classification of genetic sequences [12]. It was used in a support system for clinical decisions regarding the diagnosis of celiac disease in a survey conducted in 2011 by the Department of Pediatrics at the University of São Paulo [22]. In this study, the database consisted of 178 records and was rated by 5 artificial intelligence techniques (decision trees, Bayesian networks, nearest neighbor algorithm, support vector machines and neural networks). Among the algorithms analyzed, AODE showed the greatest accuracy (84.0), a value similar to our findings (83.0). Compared to Naive Bayes, AODE appears to be the best classifier for 
global data sets and to enable improved data characterization [23].

\section{Conclusion}

The development of this research includes the understanding that Bayesian classifiers use the Bayes theorem, which allows them to stand out because of their efficiency, speed and accuracy, thus explaining its usefulness in the diagnosis process.

Based on the results of analyzing statistical measures in the experiments described here (instances classified correctly and incorrectly, the kappa coefficient, mean absolute error, sensitivity, specificity, accuracy, recall, F-measure, and AUC), all classifiers showed good results, thus, given these data, it is possible to produce a reasonably accurate estimate of the diagnosis.

\section{Acknowledgements}

Financiadora de Estudos e Projetos (FINEP), Fundação de Amparo à Pesquisa e Inovação do Estado de Santa Catarina (FAPESC), Universidade do Extremo Sul Catarinense (UNESC). Maria Inês da Rosa e Ana Carolina Silva by the data available for the tests.

\section{Conflict of Interest}

The authors have declared no Conflict of interest.

\section{References}

1. Kanis JA, McCloskey EV, Johansson H, Cooper C, Rizzoli $\mathrm{R}$, Reginster J-Y. European guidance for the diagnosis and management of osteoporosis in postmenopausal women. Osteoporos Int 2012: 24: 23- 57.

2. Fontes MPF; Araújo LFB; Soares PRG. Osteoporose no climatério I: epidemiologia, definição, rastreio e diagnóstico. Femina 2012: 40(2): 109-116.

3. Eriksen FE. Treatment of osteopenia. Rev Endocr Metab Disord 2011: 13:209- 223.

4. Warriner HA, Saag GK. Osteoporosis Diagnosis and Medical Treatment. Orthop Phys Ther Clin N Am 2013: 44: 125-135.

5. Kaufman JM, Lapauw B, Goemaere S. Current and future treatments of osteoporosis in men. Best Pract Res Clin Endocrinol Metab 2014: 28(6): 871-884.

6. Rodrigues FA, Amaral LR. Aplicação de Métodos Computacionais de Mineração de Dados na Classificação e Seleção de Oncogenes Medidos por Microarray. Rev bras cancerol 2012: 58(2): 241249.

7. Rezende, SO. Sistemas inteligentes: fundamentos e aplicações. Barueri, SP: Manole, 2005.

8. Mello, LC. Descoberta de conhecimento em banco de dados (mineração de dados) e redes bayesianas: estado da arte, 2001.

9. Santos EB. A Ordenação das Variáveis no Processo de Otimização de Classificação Bayesianos: Uma Abordagem Evolutiva [master's thesis]. [São Carlos]: Universidade Federal de São Carlos. Curso Ciência da Computação; 2007.

10. Cruz-Ramírez N, Acosta-Mesa HG, Carrillo-Calvet H, NavaFernández LA, Barrientos-Martínez RE. Diagnosis of breast cancer using Bayesian networks: a case study. Comput Biol Med 2007: 37(11): 1553-64.

11. Friedman N, Geiger D, Goldszmidt M. Bayesian network classifiers. Mach Learn 1997: 29: 131-163.

12. Htike ZZ, Win LS. Classification of eukaryotic splice-junction genetic sequences using Averaged One-Dependence Estimators with subsumption resolution. Procedia Comput Sci 2013: 23: 36-43.

13. Martínez-Morales $M$, Cruz-Ramírez N, Jiménez-Andrade JL, Garza-Domínguez R. Bayes-N: An Algorithm for Learning Bayesian Networksfrom Data Using Local Measures of Information Gain Applied to Classification Problems. Veracruz, México: MICAl; 2004: 527-535.

14. World Health Organization. Burden of disease in DALYs by cause, sex and mortality stratum in WHO regions, estimates for 2002. Geneva: World Health Organization, 2004.

15. Abrahamsen B, Rejnmark L, Nielsen SP, Rud B, Nissen N, Mosekilde L, Bärenholdt O, Jensen JE. Ten-year prediction of osteoporosis from baseline bone mineral density: development of prognostic thresholds in healthy postmenopausal women. The Danish Osteoporosis Prevention Study. Osteoporos Int 2006: 17(2): 245-51. 
16. Ferreira D, Oliveira A, Freitas A. Applying data mining techniques to improve diagnosis in neonatal jaundice. BMC Med Inform Decis Mak 2012: 12:143.

17. Ng SYS, Xing Y, Tsui LK. A naive Bayes model for robust remaining useful life prediction of lithium-ion battery. Appl Energy 2014: 118: 114-123.

18. Li L, Zhang Q, Ding Y, Jiang H, Thiers BH, Wang JZ. Automatic diagnosis of melanoma using machine learning methods on a spectroscopic system. BMC Med Imaging 2014: 14(1): 36.

19. Wang KJ, Makond B, Wang KM. Modeling and predicting the occurrence of brain metastasis from lung cancer by Bayesian network: a case study of Taiwan. ComputBiol Med 2014: 47: 147-60.

20. Chimieski BF, Fagundes RDR. Association and Classification Data Mining Algorithms Comparison over Medical Datasets. J. Health Inform 2013: 5(2): 44-51.

21. Amorim JVM, Barone $D$, Mansur UA. Técnicas de Aprendizado de Máquina Aplicadas na Previsão de Evasão Acadêmica. XIX Simpósio Brasileiro de Informática na Educação; Nov 12-14; Fortaleza, CE. Rio de Janeiro, 2008.

22. Tenório JM, Hummel AD, Cohrs FM, Sdepanian VL, Pisa IT, de Fátima Marin $\mathrm{H}$. Artificial intelligence techniques applied to the development of a decision-support system for diagnosing celiac disease. Int J Med Inform 2011: 80(11): 793-802.

23. Flores JM, Gámez AJ, Martínez MA. Domains of competence of the semi-naive Bayesian network classifiers. Inf Sci 2014: 260: $120-148$

\section{Comment on this article:}
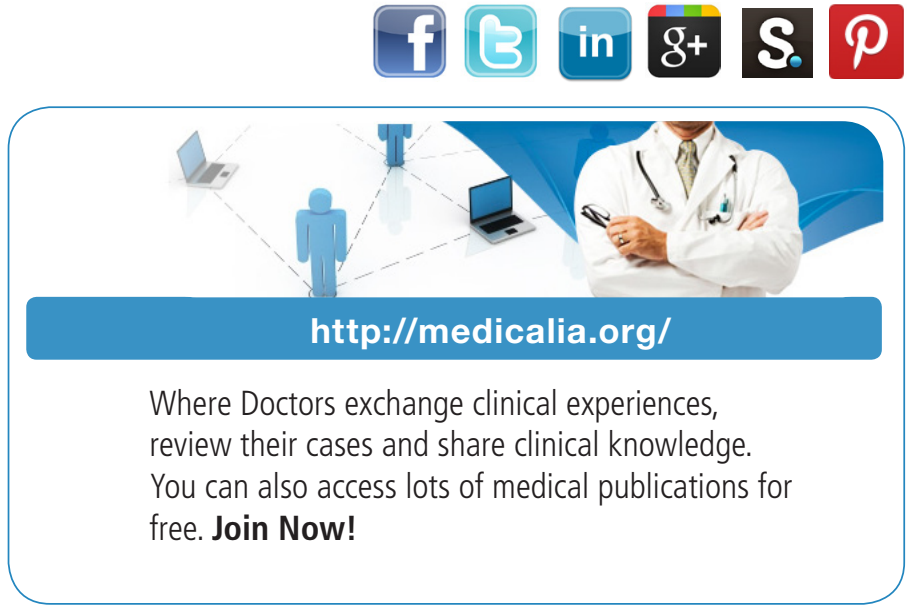

\section{Publish with iMedPub}

\section{http://www.imed.pub}

International Archives of Medicine is an open access journal publishing articles encompassing all aspects of medical science and clinical practice. IAM is considered a megajournal with independent sections on all areas of medicine. IAM is a really international journal with authors and board members from all around the world. The journal is widely indexed and classified Q1 in category Medicine. 